\title{
Fertility, Mortality and Environmental Policy
}

\author{
Ulla Lehmijoki \\ University of Helsinki \\ HECER and IZA \\ Tapio Palokangas \\ University of Helsinki \\ HECER, IZA and IIASA
}

Paper to be presented in Jekaterinburg, October 2-7, 2016, Russia 


\section{Contents}

(1) The economy as a whole

(2) Microeconomic behavior

(3) Environmental policy 


\section{Population}

- The rate of population growth, $f-m$, is the difference between the fertility rate $f$ and mortality rate $m$ :

$$
\frac{\dot{N}}{N} \doteq \frac{1}{N} \frac{d N}{d t}=f-m, \quad N(0)=N_{0},
$$

where $t$ is time and $N$ population.

- We assume that rearing each newborn requires a fixed amount $q$ of labor. Then, the number of newborns is given by $f N$, the total labor in child rearing by qfN, and labor devoted to production, $L$, is equal to population $N$ minus labor in child rearing, $q f N$ :

$$
L=N-q f N=I N .
$$




\section{Production and emissions}

- Two sectors produce the same good, clean with output $Y_{C}$ and dirty with output $Y_{d}$. That good is consumed $C$ and invested in capital $K$.

- The clean sector $Y_{c}$ does not emit at all, but dirty sector $Y_{d}$ emits in one-to-one proportion.

- The government sets the tax $x$ on emissions $Y_{d}$ and distributes the tax revenue $x Y_{d}$ to the families through a poll transfer $s$ :

$$
x Y_{d}=s N .
$$

- Capital $K$ and labor $L$ are transferable between the sectors:

$$
L \geq L_{c}+L_{d}, \quad K \geq K_{c}+K_{d},
$$

where $L_{j}$ and $K_{j}$ are labor and capital in sector $j \in\{c, d\}$. 


\section{Production}

- The sectors $j \in\{c, d\}$ have linearly homogeneous production functions

$$
Y_{j}=F^{j}\left(K_{j}, L_{j}\right), \quad F_{K}^{j}>0, \quad F_{L}^{j}>0, \quad F_{K K}^{j}<0, \quad F_{L L}^{j}<0, \quad F_{K L}^{j}>0,
$$

where the subscripts $K$ and $L$ denote the partial derivatives of the function $F^{j}$ with respect to inputs $K_{j}$ and $L_{j}$.

- We define consumption, capital and labor inputs per head:

$$
c \doteq C / N, k \doteq K / N \text { and } k_{i} \doteq K_{j} / N \text { and } l_{j} \doteq L_{j} / N \text { for } j \in\{c, d\} \text {. }
$$

- Then, we can transform the resource constraints into

$$
\begin{aligned}
& k \geq k_{c}+k_{d}, \quad 1-q f=I \geq I_{c}+l_{d}, \\
& y_{c} \doteq Y_{c} / N=F^{c}\left(k_{c}, l_{c}\right), \quad y_{d} \doteq Y_{d} / N=F^{d}\left(k_{d}, l_{d}\right) .
\end{aligned}
$$




\section{Utility}

- Capital accumulation is given by

$$
\dot{K} \doteq \frac{d K}{d t}=Y_{c}+Y_{d}+s N-x Y_{d}-C-\delta K, \quad K(0)=K_{0}
$$

where $Y_{c}+Y_{d}$ is factor revenue from production, $s N$ poll transfers, $x Y_{d}$, emission taxes, $C$ consumption and $\delta>0$ the depreciation rate of capital.

- The representative family's expected utility for $t \in[0, \infty)$ is

$$
U \doteq \int_{0}^{\infty} \frac{1}{1-\alpha}\left[c(t)^{1-\alpha}+\beta f(t)^{1-\alpha}\right] e^{-[m(t)+\rho] t} d t, \quad \alpha>0,
$$

where $c(t)$ is consumption per head, $f(t)$ fertility and $m(t)$ mortality at time $t, \alpha \neq 0$ and $\beta>0$ are constants, and $\rho>0$ the constant rate of time preference. 


\section{The representative family}

- The representative family takes the mortality rate $m$, the tax $x$ and the poll transfer $s$ as given.

- It maximizes its expected utility for the period $t \in[0, \infty)$,

$$
U \doteq \int_{0}^{\infty} \frac{1}{1-\alpha}\left[c(t)^{1-\alpha}+\beta f(t)^{1-\alpha}\right] e^{-(m+\rho) t} d t
$$

by its per capita consumption $c$ and the allocation of labor and capital, $\left(I_{c}, l_{d}, f, k_{c}, k_{d}\right)$, subject to technology $Y_{j}=F^{j}\left(K_{j}, L_{j}\right), j \in\{c, d\}$, labor supply $L=N-q f N$ and the accumulation of capital per head,

$$
\begin{aligned}
& \dot{k}=\left(\frac{\dot{K}}{N}\right)=F^{c}\left(k_{c}, l_{c}\right)+(1-x) F^{d}\left(k_{d}, l_{d}\right)+s-c+(m-f-\delta) k, \\
& k(0)=k_{0} .
\end{aligned}
$$




\section{Maximization}

- Solving this maximization problem by Pontryagin's maximum principle yields fertility $f=\varphi(k, x)$, per head revenue

$$
\begin{aligned}
& y(k, f, x) \doteq \\
& \max \\
& \begin{array}{c}
\left(I_{c}, I_{d}, k_{c}, k_{d}\right) \text { s.t. } \\
k \geq k_{c}+k_{d}, 1 \geq q f+I_{c}+I_{d}
\end{array} \\
& {\left[F^{c}\left(k_{d}, l_{d}\right)+(1-x) F^{d}\left(k_{d}, l_{d}\right)\right]}
\end{aligned}
$$

and the Euler equation

$$
\frac{\dot{c}}{c}=\frac{1}{\alpha}\left[\frac{\partial y}{\partial k}-\rho-\delta-f\right]=\frac{1}{\alpha}\left[\frac{\partial y}{\partial k}(x)-\rho-\delta-\varphi(k, x) c\right] .
$$




\section{Result}

\section{Proposition}

Assume that the tax rate $x$ is held constant. Dirty production $y_{d}$ and the fertility rate $f$ are then positively correlated, if the dirty sector is the capital-intensive sector:

$$
\frac{\partial y_{d}}{\partial f}(x)>0 \Leftrightarrow \frac{k_{d}}{l_{d}}>\frac{k_{c}}{l_{c}} .
$$




\section{Pollution}

- We assume that the mortality rate $m$ is an increasing function of total pollution $P$ :

$$
m(P), \quad m^{\prime}>0
$$

- Emissions $Y_{d}$ generate pollution $P$ according to $\dot{P} \doteq \frac{d P}{d t}=y_{d} N-\omega P=-\frac{\partial y}{\partial x} N-\omega P, \quad 0<\omega<1, \quad P(0)=P_{0}$ where the constant $\omega$ is the absorbtion by nature.

- Noting these, the rate of population growth becomes

$$
\frac{\dot{N}}{N}=\varphi(k, x) c-m(P), \quad N(0)=N_{0} .
$$




\section{Capital accumulation at the macroeconomic level}

- The dynamics of the economy is dictated by four differential equations:

- the Euler equation

- the evolution of pollution $P$

- the evolution of population $N$

- the evolution of capital per head $k$

This system has three predetermined variables, pollution $P$, population $N$ and the capital-labor ratio $k$ and per head consumption $c$ as a jump variable.

- Thus, the system must have three stable roots and one unstable root to have a saddle point solution and a unique steady-state equilibrium. This holds true if and only if

$$
\frac{\partial y}{\partial k}+\varphi_{k} \frac{c}{\varphi}>\delta .
$$




\section{Steady state}

- We denote the steady-state value of a variable by superscript $\left({ }^{*}\right)$.

- In the steady-state equilibrium $\left(c^{*}, f^{*}, k^{*}, P^{*}, N^{*}\right)$ both population and pollution are constant.

- Because the government observes the link from pollution to mortality, the social welfare function is obtained by plugging this link into the representative family's utility function as follows:

$$
U \doteq \int_{0}^{\infty} \frac{1}{1-\alpha}\left[c(t)^{1-\alpha}+\beta f(t)^{1-\alpha}\right] e^{-[m(P(t))+\rho] t} d t
$$

- Because we consider optimal taxation in the steady state $\left(f^{*}, U^{*}, k^{*}, P^{*}, N^{*}\right)$, this social welfare takes the form:

$$
U^{*}=\frac{\left(c^{*}\right)^{1-\alpha}+\beta\left(f^{*}\right)^{1-\alpha}}{(1-\alpha)\left(f^{*}+\rho\right)} .
$$




\section{Comparative statics}

Differentiating the steady-state conditions and the social welfare function with respect to the tax $x$ yields the following result:

\section{Proposition}

(a) If and only if dirty sector is relatively capital intensive (i.e. $k_{d} / l_{d}>k_{c} / l_{c}$ ), an increase in the emission tax $x$ decreases the fertility rate $f^{*}$, the mortality rate $m^{*}$ and total pollution $P^{*}$. It also increases the capital-labor ratio $k^{*}$ and per head consumption $c^{*}$.

(b) If and only if dirty sector is relatively capital intensive (i.e. $k_{d} / I_{d}>k_{c} / I_{c}$ ), and if the family's elasticity of substitution between fertility and consumption, $\frac{1}{\alpha}$, is low enough, then a small emission tax $x$ is welfare enhancing. 


\section{Interpretation}

- If the dirty sector is more capital-intensive than the clean sector, then the Rybczynski theorem implies that the environmental tax $x$ on the dirty sector rises the relative price of the this technology to $1+x$.

- This curbs down dirty production and transfers factors of production to the labor-intensive clean sector. The increasing demand for labor puts an upward pressure on wages.

- A higher wage, in turn, encourages labor to migrate from child rearing to production. This will decrease fertility and increase per head consumption. 


\section{Capital intensive clean sector}

Let us now adopt the opposite assumption that the clean sector is the more capital intensive one: $k_{d} / l_{d}<k_{c} / l_{c}$.

- Then, the emission tax $x$ increases the relative price of the dirty technology from 1 to $1+x$.

- This curbs down dirty production and transfers factors of production to the capital-intensive clean sector, putting a downward pressure in wages.

- A lower wage discourages employment and pushes people to child rearing, which increases the fertility rate and may decrease welfare in future.

- Thus, surprisingly, a subsidy to dirty production can be welfare enhancing, if $k_{d} / I_{d}<k_{c} / I_{c}$. 


\section{Conclusion}

- If the dirty sector is more capital intensive than the clean sector, then the environmental policy rises labor demand and thus wages. This tends to rise the opportunity costs of children, the demand of which decreases.

- But, if the clean sector is more capital-intensive, then pollution taxes decrease wages, generating the opposite development. In that case, the burden to provide capital to greater population will curb down per head consumption. 